\title{
Modelo explicativo del rendimiento académico en matemática en estudiantes de educación secundaria
}

\author{
Explanatory model of academic performance in mathematics in \\ high school students
}

Luis Centeno Ramírez ${ }^{1}$

1 Universidad Marcelino Champagnat, Lima Perú

\section{RESUMEN}

El objetivo general fue establecer un modelo explicativo del rendimiento académico en matemática en estudiantes mujeres de Educación Secundaria; los objetivos específicos determinaron las relaciones y la explicación de la contribución del componente cognitivo y afectivo de las actitudes, las competencias funcionales y comportamentales del desempeño laboral, y las actividades en el aula, frente al rendimiento académico. La investigación es explicativa con diseño no experimental transeccional del tipo correlacional-causal; el muestreo fue no probabilístico intencional; el grupo muestral estuvo constituido por 792 estudiantes divididos en 24 secciones de cinco grados. Los instrumentos utilizados fueron; una encuesta de opinión del alumnado sobre la actuación docente del profesorado, la escala de actitudes hacia la matemática en las enseñanzas medias y universitarias, la evaluación anual del desempeño laboral docente, el Test de Stallings, y las actas oficiales de evaluación. En la investigación se determinaron las correlaciones entre las variables de estudio, sus factores, dimensiones y componentes; además se hallaron once modelos predictores y un modelo de ecuaciones estructurales de las covariaciones que contribuyen a la explicación del rendimiento académico. Estos resultados señalan que los predictores de la opinión son: las obligaciones en clase, la valoración, el programa, la relación profesor-alumno y la evaluación, que en conjunto explican el $90 \%$; la ansiedad el $50 \%$, la confianza el $44 \%$, la motivación el $37 \%$ y el agrado explica el $70 \%$ de la variabilidad de las actitudes.

Palabras clave: Modelo explicativo, predictores, rendimiento académico, matemática.

\begin{abstract}
The research is explanatory with no transactional experimental design, correlational- causal, the overall objective was to establish a descriptive model of academic performance in mathematics in secondary school female students, the specific objectives identified relationships and explaining the contribution of the cognitive and affective component of attitudes, functional and behavioral competencies of job performance, and classroom activities, compared to academic performance. The probabilistic sampling was not intentional. The sample group consisted of 792 students divided into 24 sections of five grades. The instruments used were Survey of student opinion on the teaching performance of teachers, the scale attitudes toward mathematics in middle and university education, the annual job performance evaluation of teachers, the Stallings test, and official records of evaluation. In the research, the correlations between the study variables, their factors, dimensions and components were determined; We also found eleven predictive models and a model of structural equations of covariations that contribute to the explanation of academic performance. These results indicate that the predictors of the opinion are: the obligations in class, the assessment, the program, the teacherstudent relationship and the evaluation, which together explain $90 \%$; anxiety $50 \%$, confidence 44 $\%$, motivation $37 \%$ and liking explain $70 \%$ of the variability of attitudes.
\end{abstract}

Keywords: Explanatory model, predictors, academic achievement, mathematics.

Historial del artículo:

Recibido, 13 de mayo de 2017; aceptado, 3 de junio de 2017; disponible en línea, 30 de junio de 2017

* Egresado de la Universidad Marcelino Champagnat, y docente universitario.

Correo: Icenteno@continental.edu.pe 


\section{INTRODUCCIÓN}

Entre los cambios del proceso educativo se viene abordando el tema del rendimiento académico de los estudiantes, constituyéndose un objetivo común en las instituciones educativas, el mismo que ha sido asociado con temas de misión y visión institucional, propuesta pedagógica e institucional, planteamiento de metas educativas e indicadores al año 2021 dentro de un marco de mejora en los diversos sistemas educativos de los países iberoamericanos desde el año 2008; enfatizando en la política educativa, consideraciones metodológicas, indicadores según objetivos estratégicos para las instituciones educativas a través de propuestas a largo plazo, entre otros (Consejo Nacional de Educación [CNE], 2010).

La calidad de la educación, en ciencias y en el conocimiento matemático, reciben gran influencia de una serie de procesos que ocurren dentro y fuera del aula; al interior del aula se perciben los modelos pedagógicos utilizados, limitaciones de los estudiantes en las capacidades numéricas, ausencia de material adecuado al grado y nivel de estudio, evaluación sin estándares claros de acuerdo a la realidad nacional, desigualdad en el manejo de instrumentos de evaluación, percepción de los estudiantes hacia las diversas materias y la opinión que se tiene sobre la calidad educativa sin posibilidades de cuestionamiento o diálogo; en los aspectos externos, existen muchos procesos vinculados al rendimiento académico, la gestión de la institución educativa, la formulación de objetivos a mediano y largo plazo, falta de una propuesta educativa, la economía de los estudiantes, niveles de pobreza, rendición de cuentas, participación de los padres de familia, entre otros (The World Bank, 1999).

El proceso de aprendizaje en los diferentes niveles educativos, nacionales y extranjeros, implica el uso de la matemática como parte del conocimiento, la habilidad lógica que se aprende en la escuela, la que siempre ha tenido dificultades para el logro de los objetivos propuestos en la enseñanza a través del tiempo, tanto en la comprensión como en su uso, tal como lo señalan los diversos estudios internacionales del rendimiento, realizados por el TIMSS (Trends in International Mathematics and Science Study) desde el año 2003 al 2011; estudios dirigidos por la IEA (International Association of the Evaluation of Educational Achievement) dentro de las diversas áreas de la matemática y ciencias. La propuesta de evaluación corresponde a (a) el modelo curricular centrado en el contexto educativo, (b) la escuela-docente-aula y (c) las características de los estudiantes. Los resultados obtenidos por el Perú en el último examen muestran que se ha avanzado muy poco en las áreas de Matemática (puesto 60 de 65), Comprensión lectora (62 de 65) y Ciencias (63 de 65) (TIMMS \& PIRLS, 2011 ).
Por otro lado, el Informe del Programa Internacional para la Evaluación de Estudiantes o Informe PISA (Program for International Student Assessment) realizó diversas evaluaciones en base al análisis del rendimiento de estudiantes a partir de unos exámenes mundiales que se realizan cada tres años y que tienen como fin la valoración internacional de los alumnos. En el caso del Perú, en la evaluación PISA del año 2009, los resultantes indican que en las habilidades matemáticas se ha ocupado el puesto 63 con una media de 365 de la OCDE (Organización para la Cooperación y el Desarrollo Económicos). La correlación entre las medias por país según en PISA (2003) (como se citó en Mullis, Martin, González, y Chrostowski, 2004) que señala que los valores bajan a 0,66 y 0,79 si se excluyen a dos de los países con peores resultados; en general los países occidentales tienen un mejor desempeño en PISA, los asiáticos y europeos lo hacen mejor en TIMSS, dejando a los países sudamericanos con promedio OCDE de 421 para Chile, Argentina con 388, Brasil con 386 y Colombia con 381 (PISA, 2012).

El Perú no está exento a la problemática del bajo rendimiento académico en matemática, las evaluaciones censales realizadas por la UMC (Unidad de Medición de la Calidad Educativa) en el periodo 2006 al 2011 sobre el manejo de conceptos de símbolos y términos matemáticos, interpretación de expresiones gráficas y resolución de problemas, alcanzó en estudiantes del cuarto grado a escala nacional un $45,25 \%$ y en estudiantes del quinto grado $44,84 \%$, y en las últimas evaluaciones, los porcentajes no se han incrementado para los niveles 1 y 2 en la escala planteada para el logro de capacidades; lo cual indica que no se han desarrollado las competencia matemáticas (UMC, 2011 b).

El estudio de investigación nos lleva a establecer el problema de investigación orientado a responder la interrogante general: ¿Cuáles son los factores cognitivos y afectivos que contribuyen a la explicación del rendimiento académico en matemática en estudiantes mujeres de Educación Secundaria de una institución educativa de Huancayo?, asimismo, esto orienta a plantear el objetivo general: Establecer un modelo explicativo del rendimiento académico en matemática en estudiantes mujeres de Educación Secundaria de una institución educativa de Huancayo,; así como los específicos orientados a identificar las dimensiones del componente cognitivo, afectivo $y$ comportamental de las actitudes, y las competencias funcionales del desempeño laboral docente, que contribuyen en conjunto a la explicación del rendimiento académico en matemática en estudiantes mujeres de una institución educativa de Huancayo. Por otro lado, se establecen las hipótesis en función a determinar la existencia de un modelo explicativo del rendimiento académico en matemática. 
La metodología empleada, debido a la característica de la investigación, que tiene como objetivo responder a la interrogante sobre los factores determinantes del rendimiento académico en estudiantes mujeres de Educación Secundaria, a través de un modelo explicativo, el estudio corresponde al tipo de investigación explicativa (Hernández, Fernández y Baptista, 2010); en cuanto al diseño de la investigación, al intentar responder a las preguntas de la investigación, cumplir con los objetivos y someter la hipótesis a prueba, se ha empleado el diseño de investigación no experimental transeccional del tipo correlacional causal (Hernández et al., 2010).

Las variables de estudio se dividen en:

a) Componente cognitivo: programa, obligaciones de clase, habilidades didácticas, evaluación, relación profesor-alumno, valoración global, utilidad y confianza,

b) Componente afectivo: ansiedad, utilidad $y$ confianza,

c) Competencias funcionales de los docentes: dominio curricular, planificación y organización académica, didáctica, evaluación del aprendizaje, apoyo docente, interacción, liderazgo, relaciones interpersonales, trabajo en equipo, negociación y mediación, compromiso social, iniciativa, orientación de logro.

d) Componente conductual: rendimiento académico.

Partiendo de que esta investigación está contextualiza en la Provincia de Huancayo, Departamento de Junín, el universo estaba conformado por los estudiantes del primer al quinto grado de estudios, los que ascienden a 26545 estudiantes de acuerdo a la Unidad de Estadística Educativa del Ministerio de Educación. La población de la Institución Educativa "Nuestra Señora de Cocharcas" de Huancayo estuvo conformada por todas las alumnas del primer al quinto grado, matriculadas en el periodo escolar 2012, que ascienden a 2752 sujetos, dato proporcionado por la Secretaría Académica y corroborado en la página de ESCALE (2016). La muestra de estudio fue no probabilístico intencional, el grupo muestral de estudio estuvo constituido por 792 estudiantes del sexo, seleccionadas entre estudiantes del primer al quinto grado, matriculadas en el período académico 2016.

En cuanto a los instrumentos de recolección de datos, se emplearon:

a) Encuesta de opinión del alumnado sobre la actuación docente del profesorado del Centro Andaluz Prospectiva, Universidad de Jaén (Alfa de Cronbach $=0,937$ ).

b) Escala de actitudes hacia la matemática en las enseñanzas medias y universitarias de Elena Auzmendi Escribano - Departamento de investigación y Evaluación educativa, Universidad de Deusto, Bilbao (Alfa de Crombach =0,856).

(c) Evaluación Anual del desempeño laboral del Ministerio de Educación Nacional de la República de Colombia (Alfa de Crombach $=0,937$ ).

d) Guía y herramienta para medir el tiempo de enseñanza en clase (Test de Stallings) de Jane A. Stallings (Universidad de Nashville, Tennessee) con adaptación del Banco Mundial (Alfa de Crombach $=0,748)$.

e) Acta oficial de evaluación de Educación Básica Regular - Nivel Secundario (2016).

\section{RESULTADOS}

Los resultados de la investigación son producto de una serie de análisis estadísticos descriptivos, análisis de confiabilidad de los instrumentos, análisis de correlaciones internas de los ítems, prueba de normalidad, análisis factorial exploratorio, análisis de regresión lineal múltiple, análisis de autocorrelación del modelo, análisis de covarianzas y correlación de Spearman. Estos estudios estadísticos permitieron describir los datos en un inicio de la investigación, realizar las comparaciones de datos de acuerdo a los resultados de cada una de los datos hallados de acuerdo a los instrumentos utilizados y establecer los resultados que son las puntuaciones de correlaciones entre variables y sub variables, establecer los modelos predictores del rendimiento académico, crear los modelos de ecuaciones estructurales (MEC), ajuste del modelo final y la modelización final. Dichos resultados se resumen en:

La creación de los Modelos de Ecuaciones Estructurales (MEC) se aplicó con la finalidad de medir la eficiencia de los modelos de ecuaciones estructurales generados, se usó el valor mínimo de la X2 (CMIN) como medida del error, los grados de libertad del modelo (DF) y la

RENDAC1 1 $103.423+0.125^{*} \mathrm{ANS}+$
$0.842^{*} \mathrm{DOM}+0.35^{*} \mathrm{AGR}-0.0 \mathrm{ADM}+$
$0.224^{*} \mathrm{PLA}+0.407^{*} \mathrm{APO}-0.521^{*} \mathrm{GPER}+$
$0.44^{*} \mathrm{OBL}-0.254^{*} \mathrm{EVA}-0.212^{*} \mathrm{DID}+0.052^{*} \mathrm{UTL}$

Figura 1. Ecuación de regresión y coeficiente del modelo final del Rendimiento académico.

Nota: Modelo 11 del Rendimiento académico en matemática. ANS = Ansiedad. $D O M=$ Dominio curricular, $A G R=$ Agrado, $A D M=$ Administración de recursos, PLA = Planificación y organización académica, APO = Gestión (Apoyo), GPER = Gestión personal, $\mathrm{OBL}=$ Obligaciones en clase, EVA = Evaluación, DID = Didáctica, UTL = Utilidad.

relación de estos dos parámetros (CMIN/DF), el cual se sugiere que sea menor a tres. Además, con la finalidad de obtener un modelo suficientemente explicativo, se usó el índice de bondad de ajuste (GFI), mismo que 
debe ser mayor a 0,9 (Tanaka y Huba, 1985).

Además, basado en el modelo inicial se generaron otros en función de los índices de modificación, añadiendo relaciones que ayudaban a reducir el valor de la X2 (error del modelo) y con la pérdida mínima de grados de libertad. También, se analizó el índice comparativo de ajuste (CFI) para revisar la mejoría entre un modelo y su antecesor, aceptando un modelo sobre otro si la diferencia del CFI es mayor a 0,01 (Bentler, 1990) y se buscó mantener una medida aceptable del error de los modelos generados (RMSEA), el cual debe ser menor a 0,08 (Browne \& Cudeck, 1993).

Por otro lado, la evaluación y ajuste del modelo, una vez identificado y estimado, el siguiente paso consistió en evaluar si los datos se han ajustado al modelo citado. Así mismo, esta etapa implica la interpretación global del modelo y el contraste de las hipótesis. Para evaluar el ajuste global del modelo, se han realizado en tres niveles (a) Evaluación del modelo global, (b) Evaluación del ajuste del Modelo de Medida y (c) Evaluación de ajuste del Modelo Estructural. Asimismo, en la modelización con ecuaciones estructurales y variables latentes se utilizó el path-analysis que permite estudiar los efectos de unas variables consideradas como causales sobre otras tomadas como efectos (Batista, 2000). Se generaron nueve modelos, donde las variables que se ubican en rectángulos representan las observadas, las que están en una elipse representan las no observadas o latentes, una línea con flecha en un solo sentido indica la dependencia de una variable sobre otra $y$, finalmente, una línea con doble flecha indica correlación entre las variables (Bentler, 1990).

En cuanto al modelo explicativo del rendimiento académico; el coeficiente de regresión lineal múltiple indica al factor Ansiedad como la variable que se encuentra dentro de los once modelos predictores del Rendimiento académico en matemática. Además, El coeficiente de regresión lineal indica el mejor ajuste predictivo del Rendimiento académico en el modelo final con una constante $K=3.423$, los coeficientes de las variables predictoras para la Ansiedad es .125, en cuanto al Dominio curricular es .842, el Agrado en .065, la Administración de recursos con -.350, la Planificación y organización académica es .224, la Gestión en .470, la Gestión personal en -.521, las

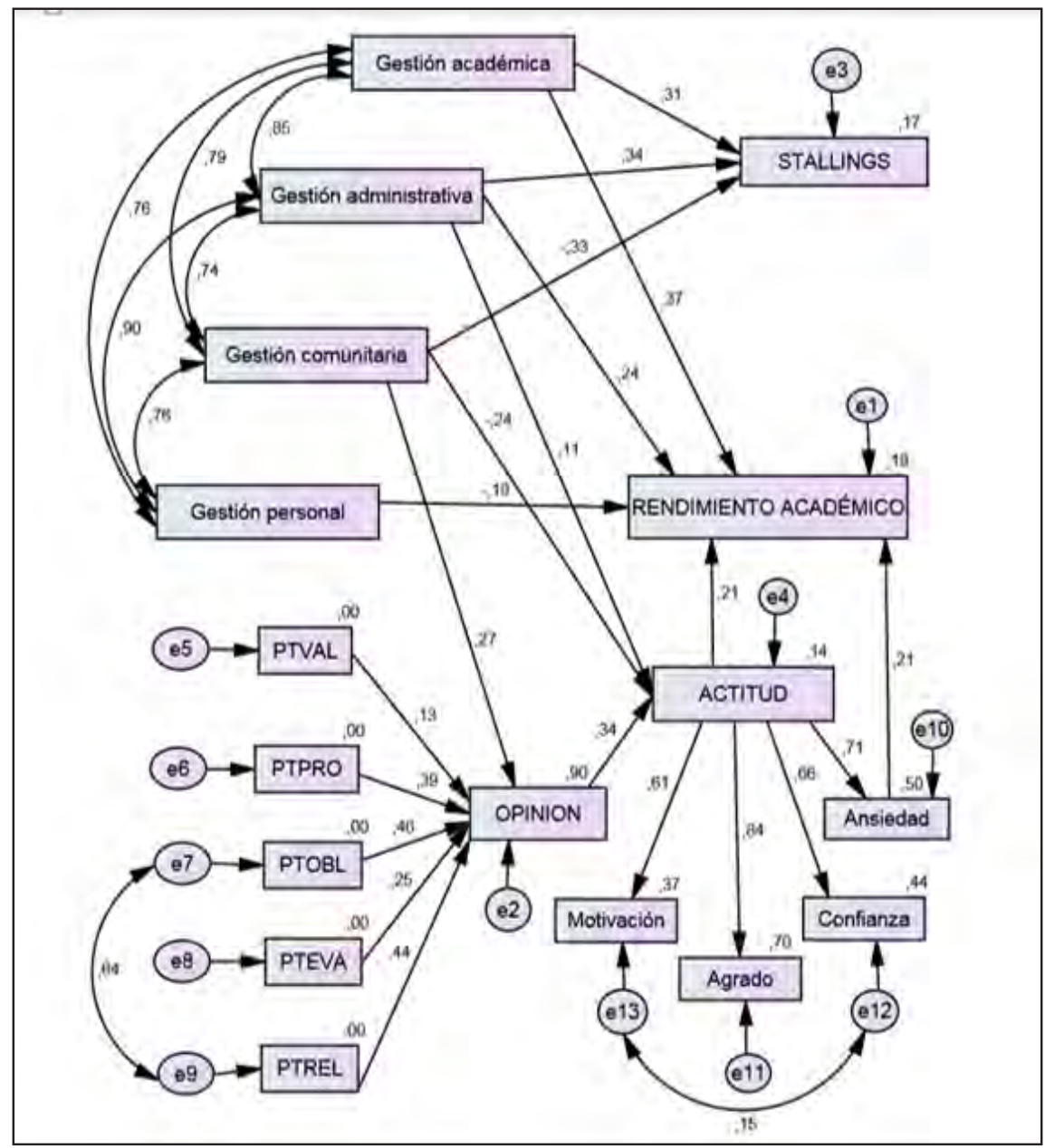

Figura 2. Modelo final de ecuaciones estructurales. 
Obligaciones en clase en .044, la Evaluación es -.254, la Didáctica en -.212 y la Utilidad en .502.

En el mismo sentido, el análisis de autocorrelación muestra que el onceavo modelo del Rendimiento académico en matemática está explicado por el 28.7 de la variabilidad de las variables predictoras en conjunto. Además, el análisis de Regresión lineal múltiple (RLM) determinó once variables predictoras del Rendimiento académico, las que corresponde a las variables predictoras Ansiedad, Agrado y Utilidad pertenecen a los factores de las Actitudes de las estudiantes hacia la matemática; en cuanto a las variables predictoras Dominio curricular, Administración de recursos, Planificación y organización académica, Gestión (Apoyo), Evaluación, Didáctica y Gestión personal pertenecen a las competencias de la Evaluación anual del Desempeño laboral; finalmente, la variable predictora Obligaciones en clase pertenece a las dimensiones de la Encuesta de opinión.

La figura 2 muestra las correlaciones de las 4 variables exógenas y las 13 relaciones que existen en las 13 variables endógenas; el índice de correlaciones directas entre las variables exógenas es .90 entre la Gestión personal con la Gestión administrativa, la Gestión administrativa se correlaciona en .85 con la Gestión académica, la Gestión personal con la Gestión académica y la Gestión comunitaria se correlacionan en .76; los menores índices de correlaciones está entre la Gestión comunitaria con la Gestión administrativa (.74). Las otras correlaciones que se hallaron, se encuentran entre la variable latente Obligaciones con Relación profesor-alumno (.64) y las variables latentes Motivación con Confianza (.15).

Asimismo, los índices de dependencia entre variables señalan que el Desempeño en aula se relaciona con la Gestión académica (.31), con la Gestión administrativa (.34) y la Gestión comunitaria (-.33). La Opinión tiene relación de dependencia de la Gestión comunitaria (.27); del mismo sentido, la Opinión influye en la Actitud (.34); como recibe influencia de la Valoración al docente (.13), Programa (.39), Obligaciones en clase (.46), Evaluación (.25) y la Relación profesoralumno (.44).

Por otro lado, la Actitud tiene una relación de dependencia frente a la Opinión (.34), y se relaciona con la Motivación (.61), Agrado (.84), Confianza (.66) y la Ansiedad (.71); además, la Ansiedad se relaciona con el Rendimiento académico (.21), y éste de manera negativa con la Gestión personal (-.18), positivamente con la Gestión administrativa (.24), la Gestión académica (.37) y las Actitudes hacia la matemática (.21). Además, se observa relaciones indirectas entre la Gestión administrativa (.11) y Gestión comunitaria (-.24) con la Actitud; a la vez, la Actitud (.21) con el Rendimiento académico; del mismo modo la Gestión comunitaria (.27) hacia la Opinión y ésta hacia la Actitud (.34) y finalmente hacia el Rendimiento académico (.21). Así mismo, la Actitud (.71) se relaciona indirectamente con la Ansiedad y la misma (.21) hacia el Rendimiento académico.

Es por ello, que de acuerdo a las variables de estudio, se pueden especificar los resultados de acuerdo a las correlaciones halladas entre las dimensiones, de la Encuesta de opinión, los factores de las actitudes hacia la matemática y el Rendimiento académico; los que se determinaron de la siguiente manera:

\section{Para el componente cognitivo}

- Existe una correlación positiva entre la Encuesta de opinión y el Rendimiento académico, mejorando sus valores promedios.

- A mayor puntuación en la dimensión Programa mejor es el índice para las Habilidades didácticas, Relación profesor-alumno, Obligaciones en clase, Evaluación y Valoración global; existiendo un buena relación entre ellas.

- A mayor puntaje total del Programa, Obligaciones en clase, habilidades didácticas, Evaluación, Relación profesor-alumno y Valoración global mayor Rendimiento académico en matemática, es decir que existe una relación positiva.

- A mayor puntuación en la dimensión Obligaciones en clase mejoran las Habilidades didácticas, el Programa, la Relación profesor-alumno, la Valoración global y la Evaluación.

- Al incrementarse las puntuaciones de la dimensión Habilidades didácticas mejora la Relación profesoralumno, las Obligaciones en clase, el Programa, la Evaluación como la Valoración global del docente.

- A mayor puntuación de la Evaluación mejora las Habilidades didácticas, la Relación profesoralumno, el Programa; las Obligaciones en clase y la Valoración global también incrementan positivamente en menor dimensión.

- A mayor puntuación de la Relación profesoralumno mejoran las Habilidades didácticas, las Obligaciones en clase y el Programa; en menor medida se incrementa la Evaluación y la Valoración global del docente.

- A mayor puntuación de la Valoración global mejoran las Habilidades didácticas, la Relación profesor-alumno, el Programa y las Obligaciones en clase.

- La relación bidireccional entre las Obligaciones en clase y la Relación profesor-alumno es alta y directa, lo que explica que a mayor Relación del profesor-alumno se mejoran las Obligaciones en clase.

- A mayor puntaje en el factor Utilidad se incrementa la puntuación de la Ansiedad, y disminuye el crecimiento del Agrado, la Confianza y la Motivación. 


\section{Para el componente afectivo}

- A mayor puntuación de la Actitud hacia la matemática mejor es el Rendimiento académico, así mismo, a mayor puntaje en la Ansiedad, Agrado, Utilidad, Motivación y Confianza, mejora el nivel del Rendimiento académico en matemática; existiendo relación directa para cada uno de los factores de las Actitudes hacia la matemática.

- Al incrementarse la puntuación del factor Ansiedad se incrementa el nivel del Rendimiento académico.

- A mayor puntuación del factor Ansiedad se incrementa el factor Utilidad y el Agrado; así mismo, en menor medida mejora la Confianza y la Motivación.

- A mayor puntuación del factor Agrado mejor es la puntuación de la Motivación, la Confianza y la Ansiedad.

- A mayor Motivación mejora el Agrado, la Confianza; disminuye el crecimiento de la Ansiedad y la Utilidad.

- Al mejorar la Confianza mejora el Agrado, la Motivación y en menor medida crece la Ansiedad y la Utilidad por la matemática.

\section{Para el componente funcional}

- A mayor Desempeño laboral mejor es el Rendimiento académico, mientras que a mayor Dominio curricular, Planificación y organización académica, Didáctica, Evaluación, Gestión (Apoyo), Administración de recursos, Convivencia, Interacción comunal y Gestión personal mejora el Rendimiento académico en matemática.

- Al mayor Dominio curricular mejor es la Gestión (Apoyo) y la Administración de recursos; así mismo, en menor crecimiento se da con el Dominio curricular, la Planificación y organización académica, la Didáctica, la Evaluación, la Convivencia, la Interacción comunal y la Gestión personal.

- Al mejorar la Didáctica mejora la Convivencia, la Gestión (Apoyo), la Planificación y organización académica, el Dominio curricular, la Gestión personal, la Evaluación, la Administración de recursos y la Interacción comunal en menor medida.

- A mayor puntuación en la Evaluación de los aprendizajes mejoran todas las competencias de la Evaluación anual del Desempeño laboral, especialmente la Administración de recursos, la Gestión personal, la Gestión (Apoyo), y la Planificación y organización académica.

- A mejor Gestión (Apoyo) mejoran las competencias de la Evaluación anual del Desempeño laboral, especialmente la Gestión personal, la Convivencia, la Evaluación, la Planificación y organización académica, la Administración de recursos, la Didáctica y el Dominio curricular.
- A mejor Convivencia y Gestión (Apoyo) mayor incremento en las competencias de la Evaluación anual del Desempeño laboral del docente de matemática.

- A mayor puntuación de la Administración de recursos mayor es la Evaluación, la Gestión personal, la Gestión (Apoyo) y el Dominio curricular; en menor medida la Planificación, la Convivencia y la Didáctica.

- A mayor valor en la Interacción comunal mejora, en menor medida, las demás competencias de la Evaluación anual del Desempeño laboral.

\section{Para las competencias comportamentales}

- La variable predictora Gestión comunitaria tiene un efecto negativo sobre la Actitud y el desempeño en aula, de manera que a menor Gestión comunitaria mayor Actitud hacia la matemática y mayor desempeño en aula.

- La variable predictora Gestión personal tiene un efecto negativo sobre el Rendimiento académico, de manera que a una menor Gestión personal mayor es el Rendimiento académico.

\section{DISCUSIÓN}

\section{Componente cognitivo}

En el modelo final de ecuaciones estructurales, se verifica que el planteamiento propuesto sobre la contribución de las dimensiones a la explicación del Rendimiento académico; la validez de $(\mathrm{H} 1)$ es dada por las covariaciones del Programa, Obligaciones de clase, Valoración global, Evaluación y Relación profesor-alumno, con la Opinión del alumnado sobre la actuación docente; la Opinión covaría con la Actitud hacia la matemática y ésta con el Rendimiento académico. El factor confianza tiene una dependencia recursiva de la Actitud y ésta sobre el Rendimiento académico en matemática. Sin embargo no es válida la hipótesis para la dimensión Habilidades didácticas y el factor Utilidad del componente cognitivo.

\section{Componente afectivo}

En el Modelo final de ecuaciones estructurales, muestra las covariaciones del componente afectivo de las actitudes hacia la matemática, donde se verifica la validez de la hipótesis $\mathrm{H} 2$ al observar la dependencia recursiva de la Motivación y el Agrado de la Actitud y ésta hacia el Rendimiento académico; en el caso de la Ansiedad, se observa la covariación de la Actitud y directamente hacia el Rendimiento académico.

Al relacionar el Rendimiento académico con los factores 
de las Actitudes hacia la matemática, se encuentra una asociación directa y medianamente estrecha con el factor Ansiedad; esto explica la predominancia del componente emocional de la ansiedad relacionada con los exámenes, la actitud aprendida y las actitudes hacia el Rendimiento académico (Reyes, 2003). La ansiedad en los estudiantes tiene correlación positiva, es decir que frente al resultado de las evaluaciones, didáctica del docente y exposición a la matemática; las referencias indican que entre el $10 \%$ y el $20 \%$ de los escolares se ven afectados por problemas relacionados con la ansiedad (Barret 1998). Además, se puede explicar el resultado de la relación existente entre la Ansiedad y el rendimiento en cuanto las mujeres suelen manifestar una mayor ansiedad hacia la matemática, dado por la inexperiencia o reflejo de una socialización pasada para evitar las áreas cuantitativas (Frank y Richard, 1988).

\section{Competencias funcionales}

En el modelo final de ecuaciones estructurales, se observan las covariancias de las variables exógenas de la competencia funcional como sus relaciones estadísticas; se acepta la validez de la hipótesis $(\mathrm{H} 3)$ al existir relación de dependencia unidireccional de la Gestión académica, que involucra los componentes Dominio curricular, Planificación y organización Didáctica y Evaluación de los aprendizajes, hacia el Rendimiento académico en matemática. Además, la variable exógena Gestión administrativa, que involucra el Uso de recursos y el Seguimiento-monitoreo, covaría con el Rendimiento académico; la competencia Gestión personal es recursiva con el Rendimiento académico. Del mismo modo, la Gestión comunitaria covaría con la Actitud y ésta con el Rendimiento académico.

El resultado del path-analysis señala relación causal directa de la Gestión académica hacia la Opinión, esto explica el efecto del (a) Dominio curricular que consiste en aplicar y enseñar los conocimiento del área, incorporando competencias y capacidades de acuerdo al plan de estudios (DCN, 2009), (b) la Planificación entendida como la organización de los procesos de enseñanza-aprendizaje en función a un proyecto, aplicando estrategias para el logro de resultados en un periodo lectivo, programar las sesiones de acuerdo a las necesidades de las estudiantes, establecer los espacios de tiempo, reglas, normas de comportamiento y los niveles de logro del aprendizaje, y (c) la Didáctica de los docentes que se basa en la capacidad de aplicar modelos pedagógicos en el diseño como en la ejecución del proceso de aprendizaje basados en teorías pedagógicas que motiven a los estudiantes alcanzar los objetivos propuestos (Ministerio de educación Nacional, 2008), direccionados hacia la variable Opinión de las estudiantes en relación al aprendizaje de la matemática, explicando la creencia que se forma hacia la matemática en relación a su dificultad o facilidad para aprender, la imagen que se forma el estudiante en cuanto juzga la matemática y la evaluación que hace hacia la asignatura involucrando sentimientos y emociones (Fishbein y Ajzen, 1975).

\section{Competencias comportamentales}

En el modelo final de ecuaciones estructurales, señala una relación recursiva entre la competencia comportamental del Desempeño laboral de los docentes (Gestión personal) y el Rendimiento académico, dando validez a la hipótesis planteada $(\mathrm{H} 4)$ sobre la contribución de la competencia comportamental con la explicación del Rendimiento académico en matemática en estudiantes mujeres de Educación Secundaria de una institución educativa de Huancayo.

La Gestión personal ejerce relación causal directa hacia el rendimiento académico; es decir, las competencias comportamentales de los docentes, como (a) las habilidades que cuentan al momento de direccionar la enseñanza-aprendizaje de la matemática basado en un enfoque por competencias y una intervención eficaz de la matemática en la vida cotidiana (Zabala, 2007), y (b) las destrezas docentes para la integración de los conocimientos en la construcción de las competencias numéricas, las cuales se forman desde la escuela hacia los diversos niveles, dominio de la materia, sus actitudes, valores, motivaciones, experiencia y la propia formación inicial docente (Pérez, Barquín \& Angulo, 1999, citados en Rodríguez, 2010).

\section{Modelo explicativo del rendimiento académico}

En base al análisis de los modelos predictores del Rendimiento académico, en función a la ecuación de regresión, se puede sostener que hay once factores diferenciales del Rendimiento académico y que existe un modelo distinto para cada uno de ellos.

El modelo final de la ERM del rendimiento académico indica que una estudiante sacaría 3.423 puntos, incrementado en .125 por la puntuación de la Ansiedad, aumenta .824 por el dominio curricular, a lo que se incrementa .65 en la valoración sobre el Agrado, disminuyendo .35 por la Administración de recursos, a ello se aumenta .224 de la Planificación, restándole .521 de la Gestión personal, más .44 de Obligaciones en clase, menos .254 de la Evaluación y .212 de la Didáctica, aumentando .052 de la Utilidad. Esto se contrasta con el modelo predictivo de Centra y Potter (1980), que sugiere que el rendimiento escolar es afectado por las características del profesor (a) cualidades, (b) experiencias, (c) dominio de la materia, y (d) la didáctica, incidiendo directamente en el rendimiento del alumno. 
En conclusión:

En el componente cognitivo, la variable exógena Obligaciones en clase es el predictor del rendimiento académico en estudiantes mujeres, con mayor efecto directo y positivo sobre la Opinión de las estudiantes, seguido por los efectos que causan las variables Valoración, Programa, la Relación profesor-alumno y la Evaluación; que en conjunto explican el $90 \%$ de la variabilidad de la Opinión.

Respecto al componente afectivo, un mejor predictor indirecto del rendimiento académico en estudiantes mujeres es el de las Actitudes a través de la Ansiedad sobre el Rendimiento académico que es inferior a los otros predictores $\left(.15=.71^{*} .21\right)$. La variable dependiente Ansiedad explica el 50\% de la variabilidad de las Actitudes hacia la matemática, la Confianza en $44 \%$ de la actitud, la Motivación en $37 \%$ y el Agrado explica el $70 \%$ de las actitudes.

En el componente funcional, la gestión académica es el predictor con mayor efecto de manera directa y positiva (.37), seguido por la variable predictora Gestión administrativa (.24) y las Actitudes (.21), explicando en conjunto el $18 \%$ sobre la variabilidad del Rendimiento académico. La variable Gestión administrativa explica la mayor influencia directa y positiva (.34) sobre el Desempeño en aula (Stallings); acompañado del efecto positivo y directo de la Gestión académica (.31), y el efecto directo y negativo de la Gestión comunitaria (-.33); que en conjunto explican el $17 \%$ de la variabilidad del Desempeño en aula.

En lo que respecta a las competencias comportamentales, la variable predictora Gestión comunitaria tiene un efecto negativo sobre la Actitud y el desempeño en aula, de manera que a menor Gestión comunitaria mayor Actitud hacia la matemática y mayor desempeño en aula. La variable predictora Gestión personal tiene un efecto negativo sobre el Rendimiento académico, de manera que a una menor Gestión personal mayor es el Rendimiento académico.

Sobre el desempeño laboral, el resultado del Pathanalysis determinó que la variable Desempeño en aula (Stallings) es explicado en $17 \%$ de la variabilidad por las variables Gestión comunitaria, Gestión administrativa y gestión académica. Los resultados señalan que no existe relación entre el Test de stallings y el Rendimiento académico; por ello, no constituye un predictor del rendimiento académico en matemática en estudiantes mujeres

Respecto al modelo explicativo del rendimiento académico, las relaciones bidireccionales (correlaciones y covarianzas) entre la Gestión académica, Gestión administrativa, Gestión comunitaria y Gestión personal, son altas y positivas, explicando que a valores altos de una de las variables exógenas hay una mayor tendencia a encontrar valores altos de la otra variable exógena. No se han hallado efectos directos o indirectos desde las variables Desempeño en aula, Gestión comunitaria y Opinión del desempeño docente sobre el Rendimiento académico, implicando una nula predicción del rendimiento académico en el rendimiento académico en estudiantes mujeres. No se han hallado relaciones entre las variables Motivación-Agrado, Motivación-Confianza y AgradoAnsiedad de las Actitudes hacia la matemática. Las variables Opinión del desempeño docente y Actitudes hacia la matemática cumplen funciones de variables predictoras latentes.

\section{REFERENCIAS BIBLIOGRÁFICAS}

Auzmendi, E. (1993). Las actitudes hacia la matemática-estadística en las enseñanzas medias y universitarias. Características y medición. Bilbao: ICE de la Universidad de Deusto. Ediciones Mensajero.

Barret, P. (1998). Evaluation of cognitive-behavioral groups treatments for Childhood Anxiety Disorders. Journal of Clinical Child Psychology, 27.

Batista, J. y Coenders, G. (2000). Modelos de Ecuaciones Estructurales. Cuadernos de Estadística 6. Madrid: La Muralla.

Bentler, M. (1990). Comparative fit indexes in structural models. Psychological Bulletin, 1990.

Browne, M., Cudeck, R. (1993). Alternative Ways of Assessing Model Fit. Testing Structural Equation Models. Newbury Park, CA: Sage.

Centra, J. y Potter, D.A. (1980). School and teacher effects: an interrelational model. Review of Educational Research, 2, 273-291.

Consejo Nacional de Educación. (2007). CNE: Proyecto Educativo Nacional al 2021. Lima: Autor.

Diseño Curricular Nacional (2009). Lima: Ministerio de Educación.

Fishbein, M., \& Ajzen, I. (1975). Belief, Attitude, Intention, and Behavior: An Introduction to Theory and Research. Reading, MA: Addison-Wesley.

Frank, M. y Richard, K. (1988). Anxiety about Research: An Initial Examination of Multidimentional Concept. Psychology of the Scientis, LVIII.

Glasman \& Biniamov (1981). An Empirical Test of an Inventory Model of Student Study Time. The Journal Economic Education, (vol. 19). Autumm, 1998.

Hernández, S., Fernández, C., \& Baptista, Z. (2010). Metodología de la Investigación. (5ª ed.). México: Mc Graw Hill. 
Ministerio de Educación Nacional (2008). Guía metodológica: Evaluación Anual de Desempeño laboral. Bogotá: Revolución educativa Colombia Aprende.

Mullis, I., Martin, M., Gonzalez, E., \& Chrostowski, S. (2004). Findings from IEA's Trends in International Mathematics and Science Study at the Fourth and Eighth Grades. Chestnut Hill, MA: TIMSS \& PIRLS International Study Center. Boston College. (Disponible en http://timss.bc.edu/timss2003i/ mathD.html).

Organismo de Estados Americanos. (2008). Eficacia Escolar y Factores Asociados en América Latina y el Caribe. OREAL/UNESCO: LLECE Laboratorio Latinoamericano de Evaluación de la Calidad de la Educación. Santiago de Chile. (Disponible en www.oei.es).

Program for International Student Assessment. (2003). PISA: Aprender para el mundo del mañana. New York: Autor. (Disponible en www.oecd.org/ pisa/39732493.pdf).

Program for International Student Assessment. (2012). PISA: Pisa in focus. New York: Autor. (Disponible en www.oecd.org/pisa/pisainfocus).

Reyes, Y. (2003). Relación entre el rendimiento académico, la ansiedad ante los exámenes, los rasgos de personalidad, el autoconcepto y la asertividad en estudiantes del primer año de psicología de la UNMSM. (Tesis de maestría). UNMSM. Lima.
Rodríguez, R. (2010). Habilidades docentes para la enseñanza de las matemáticas a nivel primaria bajo el enfoque por competencias. Memoria del 3er. Congreso Virtual Internacional de Psicólogos navegantes, vol. 6. 337 - 345 .

Tanaka, J. y Huba, G. (1985). A fit index for covariance structure models under arbitrary GLS estimation. British Journal of Mathematical and Statistical Psychology. Recuperado de www. http:// onlinelibrary.wiley.com/journal/

The World Bank (1999). Peru education at the crossroads. Challenges and opportunities for the 21 st. Century, Report № 19066-PE. Washington: The World Bank.

Trends in International Mathematics and Science Study \& Progress in International Reading Literacy Study. (2011). TIMSS \& PIRLS: International Study Center. Marcos de la evaluación: TIMMS Assessment Frameworks-Peru. Lynch School. Boston. Autor.

Unidad de Medición de la Calidad Educativa. (2011). UMC: Efecto de la escuela en el rendimiento en lógico-matemática en cuarto grado de primaria, 1. Boletín de la Unidad de Medición de la Calidad Educativa (UMC) y el Grupo de Análisis para el Desarrollo (GRADE). № 8. Lima: Ministerio de Educación.

Zabala, A. (2003). La práctica educativa. Cómo enseñar. 9a. Barcelona: Editorial Graó. 